\title{
RESEARCH
}

\section{Evaluation of efficiency in the adult cystic fibrosis clinic at the Halifax Infirmary}

\author{
Justin White MD', Pierre Landry MD, MSc'2, Meredith Chiasson MD, FRCPC ${ }^{3}$ \\ 'Department of Obstetrics and Gynecology, Dalhousie University, IWK Health Centre \\ 2Department of Medicine, Dalhousie University, QEII Health Sciences Centre \\ ${ }^{3}$ Division of Respirology, Department of Medicine, Dalhousie University, QEll Health Sciences \\ Centre
}

\begin{abstract}
The adult cystic fibrosis clinic in Halifax provides team-based care. Appointments are lengthy with high truancy. Our two-part efficiency study examined clinic flow, appointment length, and identified inefficiencies. A follow-up study was conducted to assess for improvements. Variables included total clinic time for each patient and total time spent alone waiting. Attempts to rectify problems were made after the first study. Outcomes were compared using analysis of variance. We found that patients wait significantly longer on Fridays $(p<0.05)$, residents did not negatively impact efficiency ( $>0.05$ ), and patients with known methicillin-resistant Staphylococcus aureus wait significantly longer $(p<0.05)$. Patients who arrive earlier wait significantly longer $(p<0.05)$, while patients who arrive late wait less overall. No significant difference was found after the second study. Despite changing scheduling, procedures and notifying patients, no significant improvements in efficiency were found. Further measures may be required.
\end{abstract}

$\mathrm{T}$ he adult cystic fibrosis (CF) clinic at the Halifax Infirmary (HI), in Halifax, NS, Canada, consists of a collaborative, inter-professional health care team, providing care to $140 \mathrm{CF}$ patients throughout the Maritime provinces. During a single clinic visit, a CF patient may see up to ten providers including a unit aide, respiratory therapist, physiotherapist, dietitian, social worker, psychologist, research coordinator as well as the clinic nurse, resident (or other learner) and the attending physician (respirologist).

With the median age of survival for a CF patient at 48.5 years, ${ }^{1}$ there has been a shift to a collaborative chronic care model, with a multidisciplinary team to address the social, psychological, dietary and physical realms. Provision of all necessary services through a single access point has been demonstrated to positively impact patient survival. ${ }^{2}$

As the CF clinic at our center has an interprovincial catchment area, all patients must travel to Halifax for care. Travel time, combined with the lengthy appointment required to see all ten providers represents a substantial time and financial commitment for patients. Given the complicated, multi-system course of the disease, frequent contact and aggressive therapy are felt to be necessary to maintain health and avoid decompensation. This is best done within the confines of an efficient clinic. ${ }^{3}$

In the current cost conscious environment of healthcare, increased efficiency and productivity are valued. Physicians must be increasingly productive, while balancing patient satisfaction and quality care. Up to $35 \%$ of a provider's time is spent on non-productive tasks, such as troubleshooting issues or waiting to see patients. This is frustrating not only to clinicians, but to patients, who derive no net value from these inefficient activities. ${ }^{6}$ Two commonly cited inefficiencies are bottlenecks in clinic flow, as well as time spent waiting. ${ }^{7}$

Improved efficiency and patient satisfaction need not be mutually exclusive as improved efficiency both decreases costs, and increases provider and patient satisfaction. ${ }^{3}$ Measuring clinic efficiency also allows for self-reflection regarding provider performance. Self-reflection is a valuable tool in medicine as it can modify future actions, thereby contributing to life-long learning. ${ }^{5}$

The HI is a tertiary-care teaching hospital where patients are often seen by medical students and residents, in addition to attending physicians. A study by Xakellis and Bennett examined efficiency in a family medicine resident teaching clinic. ${ }^{8}$ Patients spent on average $80.5 \pm 30$ minutes in clinic, with $27 \pm 16$ minutes accounting for time spent with the attending physician (including resident teaching). First year residents took longer to see patients compared to more senior residents, or the attending physician. Furthermore, patients who arrived on time to clinic waited significantly longer than patients who arrived late.

Gamble and Lee comment that having a resident present decreases efficiency for both the patient and 
attending physician. ${ }^{9}$ Teaching increases the time a patient spends in clinic. Time spent with learners subtracts from time which the attending physician could use to see additional patients.

This project aimed to evaluate clinic efficiency, by measuring the total appointment time, the proportion of time the patients spent waiting alone in clinic, and examining whether the presence of residents in clinics had a negative impact on either of these variables. This "alone time" represents inefficiently used time. It is hypothesized that by reallocating this time, clinic efficiency will be increased, allowing for decreased costs and improved patient and provider satisfaction, with the end goal of decreasing truancy among patients.

A similar study was undertaken in an American CF centre with the intent of increasing clinic capacity and the frequency of follow-up visits. ${ }^{3}$

\section{Methods}

A check-in and check-out chart was designed and placed on the clinic door during each clinic visit allowing each of the ten members of the CF team to record the time they entered and left the clinic room. Members where then able to extrapolate the time spent alone with each patient and their total time spent in clinic. Times were recorded using an iPhone.

The clinic visit was deemed to start when the unit aide placed the patient in the exam room and ended when the last member of the $\mathrm{CF}$ team signed out. This period represented the total time in clinic. Alone time was operationally defined as any time a patient was sitting in a clinic room alone, without any member of the CF team present. The goal of this study was not to shorten provider contact, rather to decrease lag time between each encounter, thereby decreasing non-value-added time. Smaller intervals would therefore decrease total time spent in clinic, without reducing quality of care.

There were two study periods used for data collection, June 18th to August 2nd $2013 \quad(n=55)$ and June 24th to August 1st $2014(\mathrm{n}=36)$. CF clinics were held on Tuesday afternoons and all day Friday, excluding statutory holidays. Results were recorded after each study period.

After the first study period was completed we made changes that we hoped would assist with clinic efficiency. These changes included having the methicillin resistant Staphylococcus aureus (MRSA) patients perform their spirometry in the pulmonary function lab (in the same building) and using a board to identify when a team member was in with a patient. Letters were also sent out to patients asking that they respect their clinic time. Given that changes aimed at increasing efficiency were made after the first study period, results from the second study period were compared against the first. The various conditions were subjected to a univariate analysis of variance (ANOVA) to determine statistical significance.

\section{Results}

Of the 55 patients, 26 were seen on Tuesday and 29 were seen on Friday clinics for the 2013 study period. Tuesday total clinic time (mean $=125.46$ minutes, standard deviation $[\mathrm{SD}]=9.39$ ) showed no significant difference with Friday total clinic time (mean $=140.86$ minutes, $\mathrm{SD}=8.89), \mathrm{p}=0.239$. Tuesday alone clinic time (mean=37.81 minutes, $\mathrm{SD}=7.00$ ) showed a significant difference with Friday alone clinic time (mean $=62.28$ minutes, $\mathrm{SD}=6.63, \mathrm{p}=0.014$ ).

In 2014, total clinic length was found not to be significantly different between Tuesday and Friday, with 14 patients having Tuesday appointments, and 22 on Friday. Total clinic time on Tuesday (mean $=140.43$ minutes, $\mathrm{SD}=41.36$ ) was not significantly different from Friday (mean $=166.54$ minutes, SD of 59.75, $\mathrm{p}=0.16$ ). However, time spent alone on Tuesday (mean $=35.86$ minutes, $\mathrm{SD}=21.59$ ) was significantly shorter than time spent alone on Friday (mean $=74.5$ minutes, $S D=21.59$, $\mathrm{p}=0.001$ ).

Of the 55 patients in the 2013 study period, 10 were seen by a resident. Total clinic time with a resident (mean $=152.40$ minutes, $\mathrm{SD}=15.08$ ) showed no significant difference compared to total clinic time without a resident (mean $=129.40$ minutes, $\mathrm{SD}=7.11$, $\mathrm{p}$ $=0.173)$. Alone clinic time with a resident $($ mean $=51.67$ minutes, $\mathrm{SD}=5.63)$ showed no significant difference with alone clinic time without a resident (mean $=46.40$ minutes, $\mathrm{SD}=11.93, \mathrm{p}=0.691$ ).

Similar results were found in the 2014 study period with 9 out of 36 patients receiving care from a medical resident. Total clinic time with a resident ( mean $=173.56$ minutes, $\mathrm{SD}=51.51$ ) was not significantly increased over total clinic time without a resident (mean $=150.67$ minutes, $\mathrm{SD}=54.52, \mathrm{p}=0.277$ ). Time spent alone in clinics with residents working (mean $=52.22$ minutes, $\mathrm{SD}=17.83)$, was not significantly increased in clinics without residents (mean $=59.67$ minutes, $S D=38.49$, $\mathrm{p}=0.58$ ).

Of the 55 patients, 5 were MRSA positive and 49 were MRSA negative in the 2013 study period. Total clinic time for a patient with MRSA (mean=159.33 minutes, $\mathrm{SD}=27.13$ ) showed no significant difference with total clinic time for a patient without MRSA (mean=131.02 minutes, $\mathrm{SD}=48.97, \mathrm{p}=0.167$ ). Alone clinic time for a patient with MRSA (mean=79.50 minutes, $\mathrm{SD}=14.85$ ) showed a significant difference with alone clinic time for a patient without MRSA (mean $=47.18$ minutes, $\mathrm{SD}=5.20, \mathrm{p}=0.045$ ). These results 
could not be evaluated in the 2014 study year due to only one MRSA positive patient having an appointment in the study period.

Of the 55 patients in $2013,10 / 55$ (18.2\%) of the patients arrived on time, 30/55 (54.5\%) of the patients arrived early and 15/55 (27.3\%) of the patients arrived late. It was found that being early did not significantly impact total clinic visit length $(\mathrm{p}=0.085)$, however, nor did being tardy $(\mathrm{p}=0.677)$.

The mean total clinic time with a patient who arrived on time, early or late was 121.90 minutes $(\mathrm{SD}=42.44), 146.07$ minutes $(\mathrm{SD}=51.94)$ and 116.40 minutes $(\mathrm{SD}=37.70)$, respectively. The mean alone clinic time with a patient who arrived on time, early or late was 41.90 minutes ( $\mathrm{SD}=28.95), 62.03$ minutes $(\mathrm{SD}=43.05)$, and 33.40 minutes $(\mathrm{SD}=19.66)$, respectively. Patients waited significantly longer alone in clinic if they arrived early $(\mathrm{p}<0.001)$. Patients did not wait significantly longer alone if they arrived late to clinic $(\mathrm{p}=0.871)$.

In $2014,3 / 36$ patients (8.3\%) arrived early, while $21 / 36(58.3 \%)$ arrived on time, and 11/36 (30.5\%) arrived late. Again, being early did not significantly affect total clinic length $(\mathrm{p}=0.491)$, nor did being tardy $(\mathrm{p}=0.122)$.

Those arriving on time for clinics had an average visit length of 188 minutes ( $\mathrm{SD}=46.36)$. Early arrivals waited an average of 163.43 minutes $(\mathrm{SD}=57.8)$ and late arrivals waited an average of 143.82 minutes $(\mathrm{SD}=39.62)$. The mean alone time for early, on time and late arriving patients was respectively 71.9 minutes $(\mathrm{SD}=49.44), 38.33$ minutes $(\mathrm{SD}=16.5)$ and late 49.09 minutes $(\mathrm{SD}=24.05)$. Patients arriving early did not wait significantly longer alone than those arriving late, with $\mathrm{p}=0.263$ and $\mathrm{p}=0.493$ respectively.

\section{Discussion}

This was a study of clinic efficiency for a cystic fibrosis clinic that provides care for three provinces (Nova Scotia, Prince Edward Island and New Brunswick) in Canada. Clinic efficiency is at the forefront of everyone's mind, but perhaps even more so when care is provided in a multi-disciplinary clinic. The skills that each team member provides are crucial in ensuring the best outcome for this complex patient population. As clinic times lengthen, the clinic must schedule fewer patients, resulting in patients and caregivers frustration. Prior to embarking on our efficiency study we felt that our clinic visits had elongated to the point where they were impacting on our ability to regularly see patients every three months, as recommended by current CF guidelines.
The results demonstrate six key conclusions:

1. Patients waited significantly longer alone on Friday clinics compared to Tuesday clinics in both study periods.

2. Total clinic length and time spent alone was increased in the second study period, however, providers also spent more time with each patient.

3. Residents did not contribute to clinic inefficiency in either study period.

4. MRSA positive patients waited significantly longer alone in the first study period, although this could not be evaluated in the second period due to insufficient sample size.

5. In the first study period, patients who arrived early to clinic waited significantly longer alone, compared to those arriving late. These differences were non-significant in the second part of the study. Patients who arrived early also tended to have longer total clinic visits than those arriving late.

6. Patients who arrived to clinic late tended to have shorter wait times (their total clinic time and alone time).

Our first study found that patients wait a notable amount of time alone in their clinic room. This is time that has no value added to either the patient or the health care team. Much of this down time could be attributed to the lag time between providers, the MRSA status of the patient, and whether the patient was early or late for their appointment. MRSA positive patients were booked at the end of clinic as they could not do their spirometry before a patient who was MRSA negative. If other patients arrived late for their appointment, the MRSA patient would have to wait, causing a delay in clinic flow.

We then repeated our efficiency study and were disappointed to discover that we were not significantly more efficient despite changes to clinic workflow. The number of patients studied was smaller and there were challenging logistic circumstances that arose (e.g., patient presented to clinic requesting care without an appointment, delay in out of province transportation). Gloving and gowning became standard of care between the two efficiency studies and therefore also impacted our clinic efficiency. We have not been able to see more patients in clinic, which was one of the goals of improving the clinic efficiency; but, many patients have commented that their clinic visits are shorter.

Other measures that we are considering to improve our clinic efficiency include reviewing all patient charts prior to clinic, filling out forms ahead of time for routine investigations, requesting an external evaluation of our 
clinic efficiency, and lastly, sending out letters to the patients periodically requesting that they respect their assigned clinic time.

\section{Acknowledgements}

The University Internal Medicine Research Foundation (UIMRF) provided Summer Student Research Funding for two of the authors of this paper (JW, PL). The authors would like to extend their gratitude to the cystic fibrosis clinic staff who were indispensable in the research process.

\section{References}

1. Setting the Standard Excelling in Clinical Care. (2012). Cystic Fibrosis Canada 2012 Annual Report, page 7.

2. Hofer M. Advanced chronic lung disease: need for an active interdisciplinary approach. Swiss Med Wkly 2007;137(4344):593-601.
3. Smith C, Woods S, Beauvais B. Thinking lean: implementing DMAIC methods to improve efficiency within a cystic fibrosis clinic. J Healthc Qual 2011;33(2):37-46.

4. Boffeli TJ, Thongvanh KL, Evans SJ, Ahrens CR. Patient experience and physician productivity: debunking the mythical divide at HealthPartners clinics. Perm J 2012;16(4):19-25.

5. Sandars J. The use of reflection in medical education: AMEE Guide No. 44. Med Teach 2009;31(8):685-695.

6. Murphy M. Eliminating wasteful work in hospitals improves margin, quality and culture. Murphy Leadership Institute Research Briefing: 4, 2003.

7. Sherman J. Achieving real results with Six Sigma. "Six Sigma to the rescue," declared the title of a June 2002 article in the technology section of Health Care Finance. Almost four years later, has Six Sigma helped healthcare organizations achieve the promised break- through improvement in their operations? Healthc Exec. 2006;21(1):8-10, $12-4$.

8. Xakellis GC Jr., Bennett A. Improving Clinic Efficiency of a Family Medicine Teaching Clinic. Fam Med 2001;33(7):533-8.

9. Gamble JG, Lee R. Investigating whether education of residents in a group practice increases the length of the outpatient visit. Acad Med 1991;66(8):492-3.

\title{
Diagnose ThIS
}

\section{A 25 year-old male with chest pain, fatique, and altered sensation}

\author{
Erin Martin MD, MSc \\ Division of General Surgery, University of Calgary
}

\begin{abstract}
A 25-year-old male presents to the emergency department with symptoms of generalized weakness, tingling, and numbness on the dorsum of his left hand. On further questioning, he describes a three-day history of extreme fatigue with mild dizziness, and a two-day history of intermittent chest tightness and pressure. The patient's symptoms are not triggered by exertion, as he feels mild dyspnea even at rest, but they are worsened by it. He is a 3rd year university student, admits to a fair degree of school-related stress, and states his symptoms have interfered with his ability to attend classes this week. He has a 3-year history of smoking up to half a pack of cigarettes per day, has moderate social alcohol consumption, and denies any illicit drug use. He is otherwise healthy with no known medical conditions, no previous hospitalizations or surgeries, and is not on any medications.
\end{abstract}

On examination, the patient is a tall, thin male who appears pale and anxious. His vitals are: heart rate 95 beats per minute with frequent ectopic beats palpable, blood pressure $134 / 83 \mathrm{mmHg}$, respirations 24 per minute, and oxygen-saturation $100 \%$ on room air. His speech is slow and hesitant, but he is oriented and appropriate, and easily follows commands. His cardiorespiratory exam is normal, and neurological exam is positive for an essential tremor, but negative for any focal or lateralizing findings.

Screening investigations reveal the following:

$\begin{array}{lll}\text { TEST } & \text { RESULT } & \text { NORMAL VALUES } \\ \text { RBC } & 4.8 \times 10^{12} / \mathrm{L} & 4.4-5.7 \times 10^{12} / \mathrm{L} \\ \mathrm{Hgb} & 149 \mathrm{~g} / \mathrm{L} & 140-174 \mathrm{~g} / \mathrm{L} \\ \mathrm{Hct} & 0.47 & 0.42-0.52 \\ \mathrm{Plt} & 382 \times 10^{9} / \mathrm{L} & 130-400 \times 10^{9} / \mathrm{L} \\ \mathrm{WBC} & 8.02 \times 10^{9} / \mathrm{L} & 4.0-10.0 \times 10^{9} / \mathrm{L} \\ \mathrm{Na} & 141 \mathrm{mmol} / \mathrm{L} & 135-145 \mathrm{mmol} / \mathrm{L} \\ \mathrm{K} & 4.5 \mathrm{mmol} / \mathrm{L} & 3.5-5.0 \mathrm{mmol} / \mathrm{L} \\ \mathrm{Cl} & 97 \mathrm{mmol} / \mathrm{L} & 98-106 \mathrm{mmol} / \mathrm{L} \\ \text { Urea } & 5.5 \mathrm{mmol} / \mathrm{L} & 2.5-8.0 \mathrm{mmol} / \mathrm{L} \\ \text { Creatinine } & 77 \mu \mathrm{mol} / \mathrm{L} & 70-120 \mu \mathrm{mol} / \mathrm{L} \\ \text { BGL (random) } & 5.1 \mathrm{mmol} / \mathrm{L} & <6.5 \mathrm{mmol} / \mathrm{L} \\ \text { TSH } & 2.6 \mu \mathrm{U} / \mathrm{L} & 0.4-5.0 \mu \mathrm{U} / \mathrm{L}\end{array}$

EKG 102 bpm, sinus tachycardia with PACs, normal axis, normal intervals, no blocks

Choose the most likely diagnosis after reviewing the case and investigations:
A. Arrhythmia
B. Hypoglycemia
C. Thyroid disorder
D. Hyperventilation
E. Anemia 